\title{
Comparison of the clinicopathological features and prognoses of patients with schistosomal and nonschistosomal colorectal cancer
}

\author{
ZIJIAN WANG ${ }^{1 *}$, ZHIXIANG DU ${ }^{1 *}$, YINHUA LIU $^{2 *}$, WENJIE WANG ${ }^{1}$, \\ MANMAN LIANG ${ }^{1}$, AIPING ZHANG ${ }^{1}$ and JIANGHUA YANG ${ }^{1}$ \\ ${ }^{1}$ Department of Infectious Diseases, Yijishan Hospital of Wannan Medical College; \\ ${ }^{2}$ Department of Pathology, Wannan Medical College, Wuhu, Anhui 241001, P.R. China
}

Received July 12, 2019; Accepted November 26, 2019

DOI: $10.3892 / \mathrm{ol} .2020 .11331$

\begin{abstract}
Patients with schistosomal colorectal cancer (CRC) and nonschistosomal CRC have different clinicopathological features, laboratory test results and survival rates. Long-term infection with schistosomiasis in patients with CRC may affect the pathogenesis and subsequently change the mechanisms of $\mathrm{CRC}$ in these patients, resulting in changes in the survival rates of patients with schistosomal and nonschistosomal CRC. In China, the most common type of schistosomiasis is $S$. japonicum. The present study aimed to investigate the clinicopathological features and prognostic factors of schistosomal and nonschistosomal CRC. A total of 253 patients with schistosomal CRC and 2,885 patients with nonschistosomal CRC were analyzed and their symptoms, clinicopathological features and laboratory test results were retrospectively evaluated. Patients with CRC in the present study underwent radical resection at The First Affiliated Yijishan Hospital of Wannan Medical College between January 2012 and December 2018. A total of 3,138 patients with CRC were enrolled, 253 of whom were patients with schistosomal CRC. Patients were followed-up to examine differences in the 5-year survival rates between patients with schistosomal and nonschistosomal CRC to determine whether schistosomiasis impacted the prognosis of CRC. There were significant differences in age, sex, fecal occult blood positive, pathological T stage, and CA19-9, WBC, RBC and PLT levels between patients with schistosomal CRC and nonschistosomal CRC. For residents in areas with higher levels of schistosomiasis infections, especially middle-aged
\end{abstract}

Correspondence to: Dr Jianghua Yang, Department of Infectious Diseases, Yijishan Hospital of Wannan Medical College, 2 Zheshan West Road, Wuhu, Anhui 241001, P.R. China

E-mail: yjhpath@163.com

*Contributed equally

Abbreviations: CRC, colorectal cancer; OS, overall survival

Key words: schistosomal, colorectal cancer, clinical characteristics, prognosis, survival rate and elderly males, serum tumor markers and digestive tract endoscopy should be regularly evaluated to detect the presence of digestive tract tumors as early as possible.

\section{Introduction}

Schistosomiasis is a common parasitic disease caused by parasitic flatworms (blood flukes) of the Schistosoma genus, which are highly prevalent in the Middle East, South America, parts of Southeast Asia and in sub-Saharan Africa (1,2). It is estimated that 230 million individuals worldwide are infected with schistosomiasis and it is now becoming a cause for concern in Europe, particularly in southern Europe $(3,4)$. Over the past 60 years, China has achieved considerable success in combatting this disease and the incidence and prevalence of schistosomiasis in China has dropped (5). In 2017, 37,601 individuals were infected with schistosomal disease in China compared with 54,454 individuals infected in 2016, representing a $30.95 \%$ reduction; However, multiple factors, such as an increase in the number and spread of oncomelania snails spread which affects schistosomiasis epidemics, therefore there is still a risk of a rebound in the incidence in some areas (6).

In China, colorectal cancer (CRC) is one of the top five leading causes of cancer-associated death in men and women (7). A number of risk factors for CRC in Asia have been identified, including age, sex, family history and a high body fat percentage (8). The association between schistosomiasis and $\mathrm{CRC}$ has been determined previously (9) and large epidemiological studies have demonstrated an association between CRC and schistosomiasis $(10,11)$. Several studies have suggested that long-term inflammation caused by chronic schistosomal infection is a key factor in the carcinogenic process of CRC $(12,13)$. However, there are only a few studies which have reported differences in the characteristics between patients with schistosomal and nonschistosomal CRC and the number of reported schistosomal CRC cases is low $(14,15)$. Thus, it is difficult to accurately describe the difference between schistosomal and nonschistosomal CRC.

The present study analyzed the data of patients with schistosomal and nonschistosomal CRC to determine whether there were any differences between these patients as well as the impact of schistosomiasis on the prognosis of CRC. 


\section{Patients and methods}

Patients. Ethical approval for the present study was obtained from The Ethics Committee of Yijishan Hospital and informed written consent was obtained from all patients. The present study conforms to the provisions of the Declaration of Helsinki (16). Clinical data were obtained from patients with schistosomal and nonschistosomal CRC who underwent radical resection at The First Affiliated Yijishan Hospital of Wannan Medical College between January 2012 and December 2018. The inclusion criteria were: CRC diagnoses confirmed by clinical and pathological findings. Patients with schistosomal CRC were identified by the presence of Schistosoma eggs in the intestinal tissues. Patients with other malignant tumors, such as liver cancer, lung cancer and ovarian cancer, were excluded. Among the 3,138 patients with CRC, the 253 patients with schistosomiasis had mean age of $65.32 \pm 10$ years (standard deviation) with an age range of $32-85$ years old, which was significantly higher compared with patients without schistosomiasis $(61.39 \pm 11.93$ years old $)$. The 253 schistosomal patients with CRC included 174 males and 79 females, with a male to female ratio of 2.2:1. The H\&E staining in the present study was performed by the Department of Pathology, Yijishan Hospital, with a magnification of x100 and $\mathrm{x} 400$ using a light microscope.

Methods. A retrospective analysis of clinical pathology data and laboratory test results of 3,138 patients with CRC, including 253 cases of schistosomiasis and 2,885 cases of nonschistosomal CRC was performed. Patients with CRC had an age range of 31-87 years old, with a median age of 62 years old. The male: Female ratio was 1.5. Staging of patients with CRC was based on the Tumor-Node-Metastasis staging system (17). A follow-up survey of patients with CRC between January 2012 and December 2013 was performed, including 43 patients with schistosomal CRC and 57 patients with nonschistosomal CRC. The age range of the patients followed-up was 31-85 years, with a median age of 61 years. The male: Female ratio was 2.1. The follow-up patient data were included in the retrospective analysis.

Statistical analysis. Data were analyzed using SPSS (version 20.0; IBM Corp.). Data are expressed as the frequency, percentage mean \pm standard deviation and quartile range. Patient age, laboratory test results and tumor marker levels were compared using a Student's t-test. A $\chi^{2}$ test was used to compare categorical variables. $\mathrm{P}<0.05$ was considered to indicate a statistically significant difference. The Kaplan-Meier method (18) was used to generate survival curves and patient survival time was analyzed for each variable. A comparison of survival curves was performed using a log-rank test. The univariate analysis of categorical variables was performed using the Kaplan-Meier method. Continuous variables were analyzed using Cox regression analysis. Every variable was analyzed using univariate analysis to identify all potentially important predictors and then variables with $\mathrm{P} \leq 0.20$ in the univariate analysis were included in a multivariate analysis. Due to the difference between the results in the univariate analysis, it was difficult to reflect the effectiveness of the factor in the outcome of the event. Therefore, the inclusion criteria for multivariate analysis were less stringent $(\mathrm{P} \leq 0.20)$, which effectively avoided the exclusion of potentially important variables. Clinically relevant variables that may have impacted outcomes, such as demographics, lifestyle habits, medical history, results of examination and treatment regimens. Finally, multivariate Cox regression analysis was performed to identify predictive factors for overall survival (OS). $\mathrm{P}<0.05$ was considered to indicate a statistically significant difference.

\section{Results}

Clinical characteristics. Among the 3,138 patients with CRC, the average age of patients with schistosomiasis was significantly higher compared with patients without schistosomiasis. In addition, there was a larger number of patients $>60$ years old in the schistosomal group compared with the nonschistosomal group (Fig. 1). The 253 schistosomal patients with CRC was comprised of 174 males and 79 females, with a male to female ratio of 2.2:1. Among the 2,885 patients with nonschistosomal CRC, there were 1,721 males and 1,164 females and the male to female ratio was $1.48: 1$. These results showed that there were more male patients compared with female patients in both groups and the proportion of males to females in the schistosomal group was significantly higher compared with the nonschistosomal group $\left(\chi^{2}=8.090 ; \mathrm{P}=0.004\right.$; Fig. 2$)$. Regarding the age distribution, there were significant differences between schistosomal CRC group and nonschistosomal CRC group ( $\mathrm{Z}=-4.649$; $\mathrm{P}=0.001$; Table I). Both groups primarily contained patients in the 61-70 years old group, but the schistosomal CRC group had significantly more patients in the 61-70 years old group compared with the nonschistosomal CRC group. The most common tumor location for patients in the schistosomal and nonschistosomal group was the rectum, followed by the sigmoid colon and there was no significant difference between the schistosomal and nonschistosomal group ( $\chi^{2}=5.584 ; \mathrm{P}=0.349$; Table I). In the schistosomal CRC group, the positivity rate for fecal occult blood was $63.64 \%$, which was significantly higher compared with $51.75 \%$ of the nonschistosomal CRC group ( $\mathrm{P}=0.002$; Table I).

Changes in the number of patients with CRC. The number of permanent residents in Wuhu City between January 2012 and December 2018 was assessed (Table II). These data were used to calculate the incidence of schistosomal and nonschistosomal CRC in the Wuhu area (Table III). These calculations showed that the incidence of CRC in patients with schistosomiasis in 2012 was $1.09 / 1 \times 10^{5}$ individuals, which was higher compared with $2018\left(0.91 / 1 \times 10^{5}\right)$; however, the highest incidence of CRC occurred in $2016\left(1.28 / 10^{5}\right)$. Overall, the incidence of CRC appeared to be stable. The incidence of CRC in patients without schistosomiasis in 2012 was $1.73 / 10^{6}$, which was higher compared with the incidence in $2018\left(7.9 / 10^{5}\right.$; Fig. 3). The proportion of patients with CRC in the same period was calculated for these two groups and when compared with the incidence of CRC in the two groups, the trend for the proportions was similar and the overall trend was stable.

Pathological features, routine blood tests and tumor markers. No significant difference between schistosomal and nonschistosomal groups was observed in terms of the degree of 


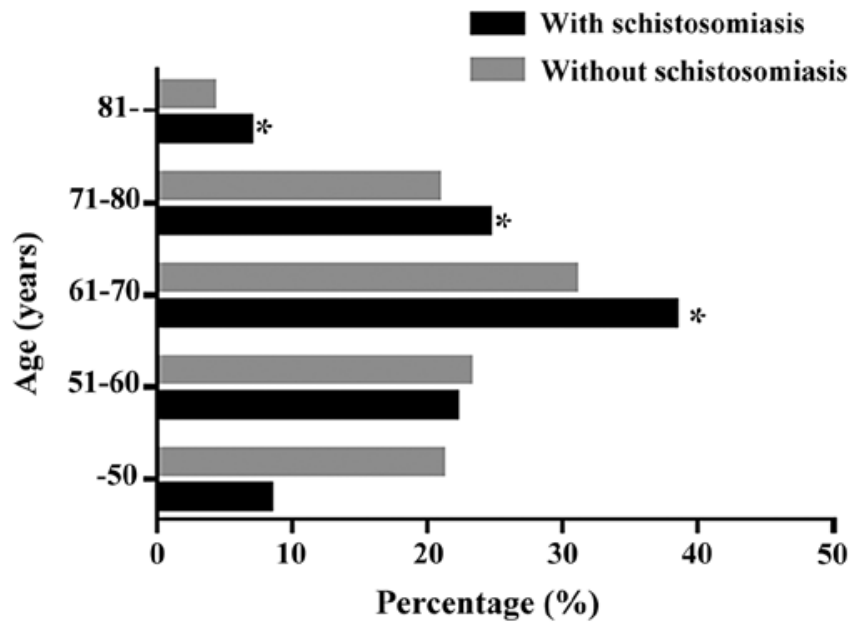

Figure 1. Percentage of patients with colorectal cancer in different age groups with and without schistosomiasis. ${ }^{*} \mathrm{P}<0.05$ vs. patients without schistosomiasis.

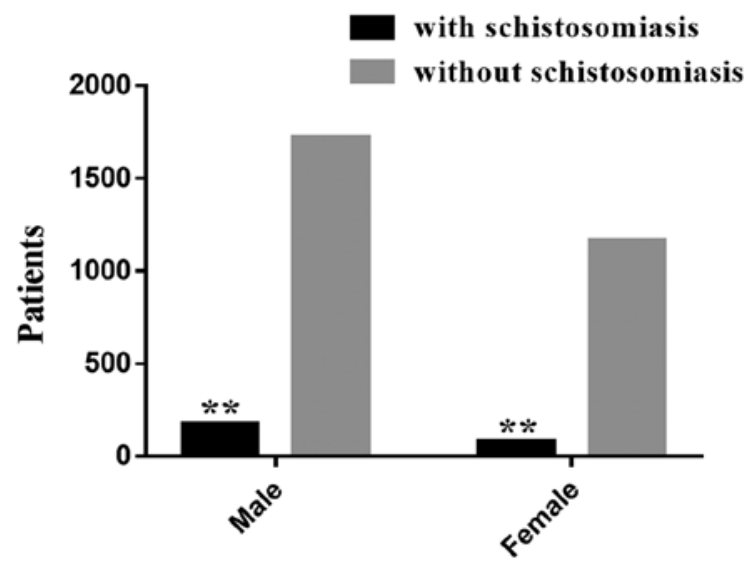

Figure 2. Distribution of male and female for patients with schistosomal CRC and nonschistosomal CRC. ${ }^{* *} \mathrm{P}<0.01$ vs. patients without schistosomiasis.

differentiation $(\mathrm{P}=0.2)$. The proportion of patients with $\mathrm{T} 1-3$ stage disease (92.24\%) compared with patients with T4 stage disease $(7.76 \%)$ was significantly higher in the schistosomal group compared with the nonschistosomal group (stage T1-3, 77.08\%; stage T4, 22.92\%; Fig. 4). The number of white blood cells (WBCs) in both groups was within the normal range $\left(4-10 \times 10^{9} / 1\right)(19)$, but the red blood cell (RBC) count in the nonschistosomal CRC group was higher compared with the schistosomal CRC group. The number of RBCs in the two groups was slightly lower compared with normal values (male,

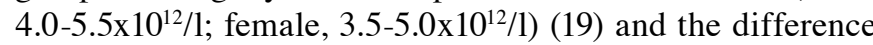
between the observed values and the reference values was statistically significant $(\mathrm{P}=0.01)$. The hemoglobin $(\mathrm{Hb})$ level in both groups was lower compared with normal values (male, 120-160 g/l; female, 110-150 g/l) (19) and there was no significant difference between the observed values and the reference values $(\mathrm{P}=0.18)$. The platelet (PLT) count in both groups was within the normal range $\left(1-3 \times 10^{11} / 1\right)(19)$. The levels of serum CEA, CA-125 and CA19-9 in the two groups were obtained from the patients' medical records. The levels of CEA and CA19-9 in patients with schistosomal and nonschistosomal
CRC were significantly higher compared with normal values (CEA reference value, <5.0 ng/l; CA19-9 reference value, 0-40 kU/l) (20), but there was no significant increase in CA-125 (normal reference value: $<35 \mathrm{kU} / \mathrm{l}$ ) (21) in either group. The level of CA19-9 was significantly higher in the nonschistosomal CRC group compared with the schistosomal CRC group ( $\mathrm{P}=0.023$; Fig. 5; Table IV).

Survival time comparison. A follow-up survey of patients with CRC between January 2012 and December 2013 included 43 patients with schistosomal CRC and 57 patients with nonschistosomal CRC. The median follow-up time was 78 months (16-90 months). The 5-year survival rate was $68.9 \%$ for the schistosomal group and $46.4 \%$ for the nonschistosomal CRC group. The 5-year survival rate of the schistosomal group was significantly higher compared with the nonschistosomal group and there was a significant difference between the observed and reference values ( $\mathrm{P}=0.026$; Fig. 6$)$. The mean age of death in the schistosomal group was $66.33 \pm 3.083$ years, which was higher compared with the nonschistosomal group $(56.29 \pm 1.943)$ and a significant difference was identified between the observed values and the reference value $(\mathrm{P}=0.0073$; Table V).

Univariate and multivariate analysis of the prognostic factors for overall survival. Univariate analysis was performed on the 100 patients used for follow-up. In the schistosomal group, the $\mathrm{T}$ stage was significantly associated with $\mathrm{OS}(\mathrm{P}=0.011)$. In the nonschistosomal group, the T stage and CA19-9 were significantly associated with $\mathrm{OS}(\mathrm{P}=0.016$ and $\mathrm{P}=0.01$, respectively). Multivariate analysis was performed to assess the prognostic value of clinicopathological factors with a statistically significant probability of being associated with $\mathrm{OS}(\mathrm{P} \leq 0.2$ in the univariate analysis; Table VI). In the schistosomal group, $\mathrm{T}$ stage and CA19-9 were independent prognostic factors for OS $(\mathrm{P}=0.029$ and $\mathrm{P}=0.032$, respectively). In the nonschistosomal group, $\mathrm{T}$ stage and CA19-9 were independent prognostic factors for OS $(\mathrm{P}=0.034$ and $\mathrm{P}=0.047$, respectively; Table VI).

\section{Discussion}

Schistosomiasis is endemic in China; however, as the prevention and control of schistosomiasis in China has improved, the overall schistosomal epidemic has declined $(22,23)$. Although progress has been made in the prevention and treatment of schistosomiasis, numerous new cases are reported every year $(24,25)$. Schistosomiasis is an infectious disease mediated by immunity (26). Following infection with schistosomiasis, the body's immune response goes through at least three processes. In the first 3-5 weeks following infection, larvae migrate into the body and the primary immune response is mediated by Th1 cells; subsequently, as the larva matures, in weeks 5-6, the male and female adults begin to lay eggs after mating. At this stage, the immune response changes significantly. The immune response mediated primarily by egg antigens significantly reduces the Th1 component and is accompanied by a strong Th2 response. Finally, the chronic infection stage is initiated (27). Th2 cells serve a key regulatory role in mediating the immune response, reducing and maintaining the volume of granulomas around the eggs (28). 
Table I. Clinical characteristics of patients with colorectal cancer.

\begin{tabular}{lcc}
\hline Variable & With schistosomiasis, $n(\%)$ & Without schistosomiasis \\
\hline Age, mean \pm standard deviation, years & $65.32 \pm 10.57$ & $61.39 \pm 11.93$ \\
$0-50$ & $21(8.3)$ & $609(21.1)$ \\
$51-60$ & $56(22.1)$ & $667(23.1)$ \\
$61-70$ & $97(38.3)$ & $891(30.9)$ \\
$71-80$ & $62(24.5)$ & $601(20.8)$ \\
$\geq 81$ & $17(6.8)$ & $117(4.1)$ \\
Sex & & 1721 \\
Male & 174 & 1164 \\
Female & 79 & $0.001^{\mathrm{c}}$ \\
Location & & $1395(48.37)$ \\
Rectum & $130(51.32)$ & $607(21.04)$ \\
Sigmoid colon & $47(18.49)$ & $106(3.65)$ \\
Descending colon & $23(9.06)$ & $126(4.38)$ \\
Transverse colon & $6(2.26)$ & 0.349 \\
Ascending colon & $13(5.28)$ & $430(14.90)$ \\
Ileocecal colon & $34(13.59)$ & $221(7.66)$ \\
Fecal occult blood positive & $161(63.64)$ & $1493(51.75)$ \\
\hline P & &
\end{tabular}

${ }^{\mathrm{a}} \mathrm{P}<0.05,{ }^{\mathrm{b}} \mathrm{P}<0.01,{ }^{\mathrm{c}} \mathrm{P}<0.001$.

Table II. Number of patients with CRC and total patients admitted for gastrointestinal surgery at Yijishan Hospital in the past 7 years.

\begin{tabular}{|c|c|c|c|c|c|c|c|}
\hline Type of CRC & 2012 & 2013 & 2014 & 2015 & 2016 & 2017 & 2018 \\
\hline CRC.SJ & 39 & 30 & 32 & 32 & 47 & 39 & 34 \\
\hline CRC.NSJ & 619 & 361 & 398 & 423 & 379 & 409 & 296 \\
\hline Total no. patients with CRC & 658 & 391 & 430 & 455 & 426 & 448 & 330 \\
\hline Total patients admitted for gastrointestinal surgery & 6,005 & 6,273 & 6,487 & 7,123 & 6,918 & 7,517 & 7,823 \\
\hline Resident population of Wuhu, $\times 10^{4}$ & 357.8 & 359.6 & 361.7 & 365.4 & 367 & 369.6 & 374.8 \\
\hline
\end{tabular}

CRC, colorectal cancer; SJ, Schistosoma japonicum; NSJ, without Schistosoma japonicum.

Table III. Incidence of $\mathrm{CRC}^{\mathrm{a}}$ by year in WuHu city ${ }^{\mathrm{a}}$.

\begin{tabular}{lrrrrrr}
\hline Type of CRC & 2012 & 2013 & 2014 & 2015 & 2016 & 2017 \\
\hline With Schistosomiasis & 1.09 & 0.83 & 0.88 & 0.87 & 1.28 & 1.06 \\
Non Schistosomiasis & 17.30 & 10.04 & 11.00 & 11.58 & 10.33 & 11.07 \\
Total & 18.39 & 10.87 & 11.88 & 12.45 & 11.61 & 12.13
\end{tabular}

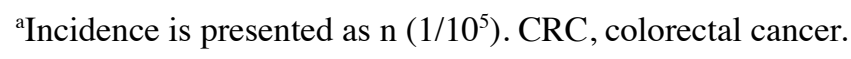

Granuloma of the liver and intestine caused by the schistosomal egg antigen and the consequent fibrosis and hardening of these tissues are the primary pathologies of patients with chronic and advanced schistosomiasis $(29,30)$. The resulting portal hypertension, intestinal obstruction and CRC are the primary causes of death in patients with advanced schistosomiasis (4).
Chronic schistosomiasis is also associated with CRC, liver cancer and bladder cancer (31-34).

The present study collected data on patients with schistosomal CRC at The First Affiliated Yijishan Hospital of Wannan Medical College for 7 years. It was found that there were patients with schistosomal CRC with a long history 
A



B

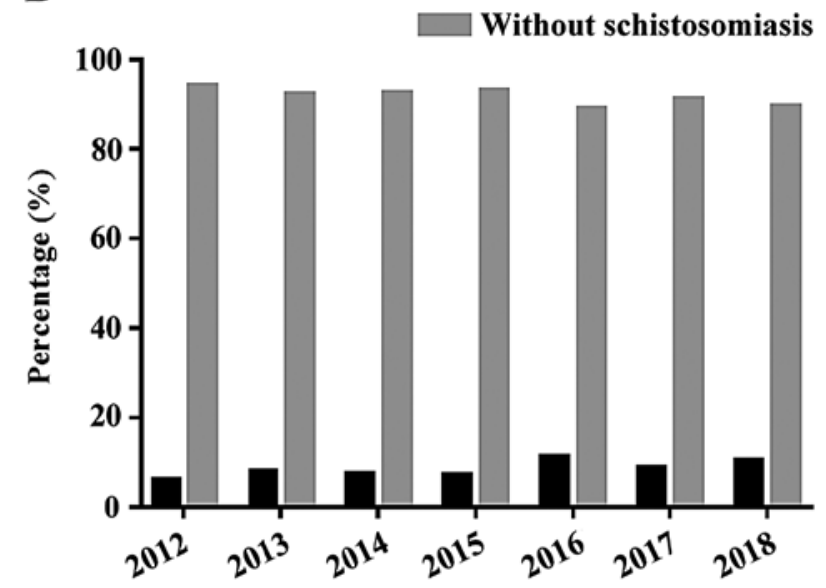

Figure 3. Incidence of CRC by year and the proportion of patients with CRC with and without schistosomiasis. (A) Incidence of schistosomal and nonschistosomal CRC by year. (B) Percentage of patients with CRC with and without schistosomiasis each year. CRC; colorectal cancer.

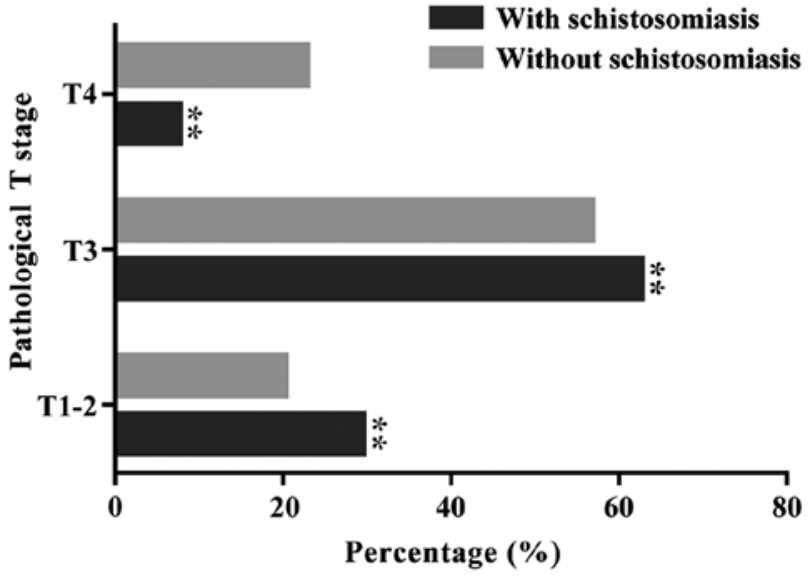

Figure 4. Distribution of patients with various T stages of colorectal cancer with and without schistosomiasis. T, tumor stage. ${ }^{* *} \mathrm{P}<0.01$ vs. patients without schistosomiasis.

of schistosomiasis infection, which is considered chronic schistosomiasis. Malignant tumors are characterized by a short disease course, rapid progression and low survival rates (24). Additionally, patients with schistosomiasis have longer medical records associated with the disease compared with patients with CRC. Patients with schistosomiasis CRC in the present study first presented with schistosomiasis and later developed CRC. It was found that although the incidence of schistosomiasis in patients with CRC has fluctuated, the overall rate of infection has remained relatively stable. This finding reflects the effort made in the prevention and control of schistosomiasis in China. In the past decade, the incidence of CRC in China has increased in the aged population. The highest incidence of CRC is in individuals $>50$ years old (8). The present study demonstrated that the percentage of patients with schistosomal and nonschistosomal CRC in patients $>50$ years old was $56.88 \%$. This finding is consistent with previously reported data in China (35). The incidence of CRC in developed countries is significantly higher compared with developing countries, which is associated with factors such as high caloric intake (36) and higher obesity rates (35). Several

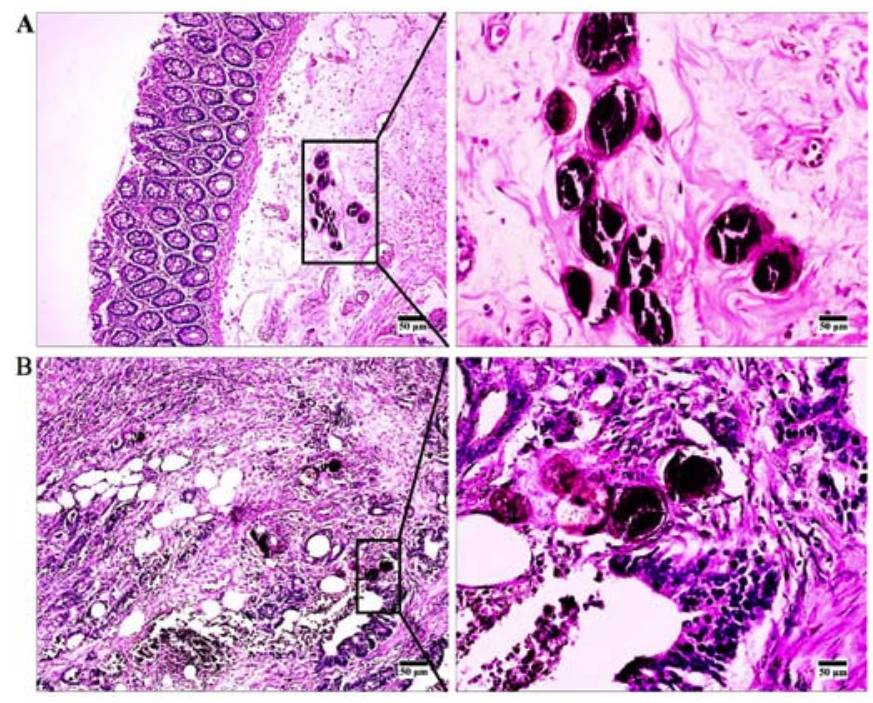

Figure 5. Histopathological features of schistosomal colorectal cancer (A) Schistosomal ova in tumor adjacent tissues. Magnification, x100 or x400. (B) Schistosomal ova in tumor tissues. Magnification, $\mathrm{x} 100$ or $\mathrm{x} 400$.

recent studies have suggested that the occurrence of $\mathrm{CRC}$ is associated with certain pathogen infections, such as infections with bacteria $(37,38)$, parasites $(15,39)$ and other infection sources, such as human bocavirus (40).

Patients with schistosomal and nonschistosomal CRC have significant differences in terms of clinicopathological features and laboratory test results, such as CEA, CA19-9 and AC-125 plasma levels (41). The present study suggested that the schistosomal CRC group had a different sex-to-age ratio compared with the nonschistosomal CRC group, which may be associated with the unique epidemiological characteristics of schistosomiasis, such as exposure to water and pathogenesis differences in males (42). It has been reported that the difference in the proportion of males and females with CRC may be associated with the dietary differences between the sexes, as females consume more fiber compared with males (42).

Regarding the differentiation of CRC, the majority of patients in the schistosomal and nonschistosomal groups 
Table IV. Pathological features, laboratory test results and tumor markers of patients with schistosomal and nonschistosomal colorectal cancer.

\begin{tabular}{lcc}
\hline Variable & $\begin{array}{c}\text { With schistosomiasis, } \\
\mathrm{n}(\%) \text { or mean } \pm \mathrm{SD}\end{array}$ & $\begin{array}{c}\text { Without schistosomiasis, } \\
\mathrm{n}(\%) \text { or mean } \pm \text { SD }\end{array}$ \\
\hline Differentiation & $7(2.64)$ & $633(21.95)$ \\
Poor & $238(93.96)$ & $2187(75.80)$ \\
Moderate & $8(3.40)$ & $65(2.25)$ \\
Well & & \\
T stage & $75(29.6)$ & $585(20.28)$ \\
T1-2 & $158(62.64)$ & $1639(56.80)$ \\
T3 & $20(7.76)$ & $661(22.92)$ \\
T4 & & \\
Tumor marker & $45.30 \pm 198.83$ & $44.68 \pm 175.74$ \\
CEA & $23.56 \pm 45.22$ & $35.39 \pm 79.31$ \\
CA-125 & $92.44 \pm 250.44$ & $115.09 \pm 281.13$ \\
CA19-9 & & \\
Blood counts & $5.87 \pm 2.40$ & $6.27 \pm 2.37$ \\
WBC, $10^{9} / 1$ & $3.79 \pm 0.62$ & $3.93 \pm 0.65$ \\
RBC, $10^{12} / 1$ & $109.47 \pm 21.75$ & $111.15 \pm 24.44$ \\
Hb, g/l & $173.45 \pm 72.89$ & $190.79 \pm 86.81$ \\
PLT, $10^{9} / 1$ & & 0.371 \\
\hline
\end{tabular}

${ }^{\mathrm{a}} \mathrm{P}<0.05,{ }^{\mathrm{b}} \mathrm{P} \leq 0.01,{ }^{\mathrm{c}} \mathrm{P} \leq 0.001$. CRC, colorectal cancer; $\mathrm{T}$, tumor; WBC, white blood cell; RBC, red blood cell; Hb, hemoglobin; PLT, platelets.

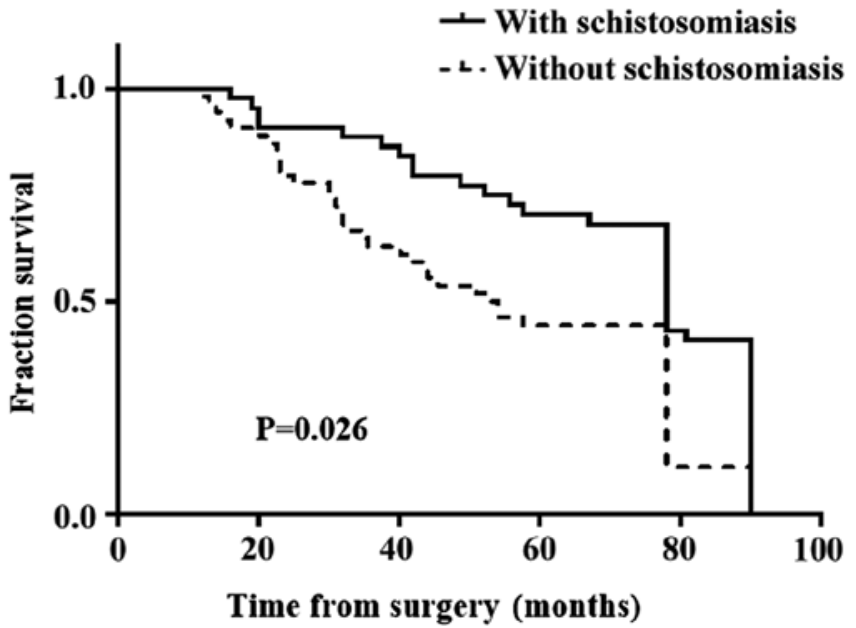

Figure 6. Survival curve of patients with schistosomal and nonschistosomal colorectal cancer. The survival rate was significantly higher in patients with colorectal cancer with schistosomiasis compared with patients without schistosomiasis.

exhibited moderate differentiation and there was no significant difference between the observed and the reference values, which is consistent with previously reported data (41). The patients in the schistosomal group were primarily in the T1-2 and T3 stages, which was significantly different from the T1-2 and T3 stage distributions in the nonschistosomal group. These results showed that the degree of malignancy in patients with schistosomal CRC was significantly lower compared with patients with nonschistosomal CRC. This finding may be due to the role that schistosomiasis serves in altering the mechanisms underlying CRC development. In the present study, serum CEA, CA-125 and CA19-9 levels were compared with normal reference values. The levels of CEA and CA19-9 in patients with schistosomal and nonschistosomal CRC were significantly higher compared with normal reference values. Moreover, the level of CA19-9 in the nonschistosomal CRC group was higher compared with the schistosomal CRC group. These results suggest that CEA and CA19-9 have a diagnostic value in CRC and that the change in CA19-9 serum levels was more prominent in simple intestinal cancer. Pengjun et al (43) reported that testing using three serum cytokines, CEA and CA19-9 may have a strong potential in aiding CRC detection. Tumor markers are useful for predicting the prognosis of patients with CRC, where CA19-9 and CEA have independent prognostic value in CRC as elevated levels are associated with a less favorable prognosis $(44,45)$.

In the present study, there were significant differences in the levels of WBCs, RBCs and PLTs between the observed and reference values. The $\mathrm{Hb}$ levels of the schistosomal and nonschistosomal group were lower compared with normal reference values, but the difference was not significant. For residents of schistosomal-endemic areas, routine blood tests, stool occult blood tests and serum tumor marker tests are thus recommended. For patients $>50$ years old (especially those who are $>60$ years, male and have unexplained anemia), early digestive tract endoscopy and other examinations are recommended to exclude the possibility of digestive tract tumors (41). This is important for the early detection of 
Table V. Follow-up of patients with colorectal cancer.

\begin{tabular}{lccc}
\hline Variable & With schistosomiasis, $\mathrm{n}=43$ & Without schistosomiasis, $\mathrm{n}=57$ & $\mathrm{P}-\mathrm{value}$ \\
\hline Deaths, $\mathrm{n}$ & 18 & 21 & - \\
Age, years, mean \pm standard deviation & $66.33 \pm 3.083$ & $56.29 \pm 1.943$ & $0.0073^{\mathrm{b}}$ \\
5-year survival & $68.90 \%$ & $46.40 \%$ & $0.026^{\mathrm{a}}$ \\
\hline
\end{tabular}

${ }^{\mathrm{a}} \mathrm{P}<0.05,{ }^{\mathrm{b}} \mathrm{P}<0.01$.

Table VI. Univariate and multivariate analysis of the prognostic factors for overall survival of patients with schistosomal and nonschistosomal colorectal cancer.

\begin{tabular}{|c|c|c|c|c|c|c|}
\hline \multirow[b]{3}{*}{ Variable } & \multicolumn{2}{|c|}{ Univariate analysis } & \multicolumn{4}{|c|}{ Multivariate analysis } \\
\hline & \multirow{2}{*}{$\frac{\text { Schistosomal }}{\text { P-value }}$} & \multirow{2}{*}{$\frac{\text { Nonschistosomal }}{\text { P-value }}$} & \multicolumn{2}{|c|}{ Schistosomal } & \multicolumn{2}{|c|}{ Nonschistosomal } \\
\hline & & & HR $(95 \% \mathrm{CI})$ & P-value & $\operatorname{HR}(95 \% \mathrm{CI})$ & P-value \\
\hline Age, years & 0.068 & 0.741 & - & 0.543 & - & - \\
\hline Sex & 0.574 & 0.861 & - & - & - & - \\
\hline Location & 0.823 & 0.71 & - & - & - & - \\
\hline Differentiation & 0.155 & 0.176 & - & 0.761 & - & 0.728 \\
\hline T stage & $0.011^{\mathrm{a}}$ & $0.016^{\mathrm{a}}$ & - & $0.029^{\mathrm{a}}$ & - & $0.034^{\mathrm{a}}$ \\
\hline $\mathrm{T} 1-2$ & - & - & $0.477(0.092-2.473)$ & & $0.225(0.057-0.892)$ & - \\
\hline $\mathrm{T} 3$ & - & - & $1.191(0.139-10.206)$ & & $0.157(0.044-0.561)$ & - \\
\hline $\mathrm{T} 4$ & - & - & $3.555(1.155-10.941)$ & & $0.633(0.204-1.962)$ & - \\
\hline \multicolumn{7}{|l|}{ Tumor marker } \\
\hline CEA & 0.372 & 0.317 & - & 0.299 & - & - \\
\hline CA-125 & 0.426 & 0.396 & - & - & - & - \\
\hline CA19-9 & 0.171 & $0.010^{\mathrm{b}}$ & $1.004(0.152-1.008)$ & $0.032^{\mathrm{a}}$ & $1.013(1-1.027)$ & $0.047^{\mathrm{a}}$ \\
\hline \multicolumn{7}{|l|}{ Blood test } \\
\hline WBC, $10^{9} / 1$ & 0.506 & 0.867 & - & - & - & - \\
\hline $\mathrm{RBC}, 10^{12} / 1$ & 0.231 & 0.435 & - & - & - & - \\
\hline $\mathrm{Hb}, \mathrm{g} / \mathrm{l}$ & 0.453 & 0.464 & - & - & - & - \\
\hline PLT, $10^{9} / 1$ & 0.907 & 0.109 & - & - & - & 0.437 \\
\hline
\end{tabular}

${ }^{\mathrm{a}} \mathrm{P}<0.05$, ${ }^{\mathrm{P}} \leq 0.01$. HR, hazard ratio; CI, confidence interval; $\mathrm{T}$, tumor stage; WBC, white blood cell; RBC, red blood cell; Hb, hemoglobin; PLT, platelets.

gastrointestinal tumors in patients and for the improvement of survival rates.

One limitation of the present study was the collected data may only be reflective of the level of CRC in the Wuhu area and thus biases may exist.

In the present study, a large quantity of patient data was collected and the prognosis of patients was analyzed. The sample size was large and the analysis was comprehensive. Based on a follow-up, the 5-year survival rate of patients with schistosomal CRC was significantly higher compared with patients with nonschistosomal CRC; however, these results differ from previously published data. A previous study analyzing 430 cases of schistosomal CRC demonstrated a 5 -year survival rate of $45.6 \%$, which was lower compared with patients without schistosomal disease $(50.9 \%$ of 2,717 patients).
The T stage and CA19-9 levels were independent prognostic factors of survival (46). In addition, Wang et al (47) analyzed 30 patients with schistosomal rectal cancer and showed that schistosomiasis was significantly associated with OS and schistosomiasis was an independent prognostic factor which predicted a less favorable disease-free survival and OS based on multivariate analysis.

In the present study, clinical and pathological features, laboratory test results and prognostic factors of schistosomal and nonschistosomal CRC were analyzed and it was found that there were significant differences between the groups. The average age of the patients with schistosomal CRC was greater compared with patients with nonschistosomal CRC and patients with schistosomal CRC primarily had T1-3 stage disease, with more patients in the early and intermediate 
stages. The 5-year survival rate of patients with schistosomal CRC was greater compared with patients with nonschistosomal CRC. Schistosomiasis serves a role in the development of CRC, particularly in the early and intermediate stages (48). The results of the present study demonstrate that patients with schistosomal CRC have a more favorable prognosis compared with patients with nonschistosomal CRC and that schistosomal infection may alter the mechanisms underlying the progression of CRC.

\section{Acknowledgements}

Not applicable.

\section{Funding}

The present study was supported by grants from The National Natural Science Foundation of China (grant nos. 30700694 and 81141083) and The Key University Science Research Project of Anhui Province, WuHu city (grant no. KJ2014A271).

\section{Availability of data and materials}

The datasets used during the present study are available from the corresponding author upon reasonable request.

\section{Authors' contributions}

JY designed the study. ZW and ZD analyzed the data and wrote the initial draft of the manuscript. YL collected the patients data and analyzed the data. WW, ML and AZ performed the statistical analysis. All authors read and approved the final manuscript.

\section{Ethical approval and consent to participate}

The present study was approved by The First Affiliated Yijishan Hospital of Wannan Medical College (Wuhu, China) and it conforms to the provisions of the Declaration of Helsinki. Written informed consent was obtained from all individuals in the present study.

\section{Patient consent for publication}

Not applicable.

\section{Competing interests}

The authors declare that they have no competing interests.

\section{References}

1. McManus DP, Dunne DW, Sacko M, Utzinger J, Vennervald BJ and Zhou XN: Schistosomiasis. Nat Rev Dis Primers 4: 13, 2018.

2. Muhubiri K, John CM and Samson M: Efficacy of praziquantel treatment regimens in pre-school and school aged children infected with schistosomiasis in sub-Saharan Africa: A systematic review. Infect Dis Poverty 7: 73, 2018.

3. Chitsulo L, Engels D, Montresor A and Savioli L: The global status of schistosomiasis and its control. Acta Trop 77: 41-51, 2000.

4. Colley DG, Bustinduy AL, Secor WE and King CH: Human schistosomiasis. Lancet 368: 2253-2264, 2014.
5. Yang Y, Zhou YB, Song XX, Li SZ, Zhong B, Wang TP, Bergquist R, Zhou XN and Jiang QW: Integrated control strategy of schistosomiasis in the People's Republic of China: Projects involving agriculture, water conservancy, forestry, sanitation and environmental modification. Adv Parasitol 92: 237-268, 2016.

6. Li-Juan Z, Zhi-Min X, Ying-Jun Q, Hui D, Shan L, Jing X, Shi-Zhu L and Xiao-Nong Z: Endemic status of schistosomiasis in People's Republic of China in 2016. Zhongguo Xue Xi Chong Bing Fang Zhi Za Zhi 29: 669-677, 2017 (In Chinese)

7. Pang Y, Kartsonaki C, Guo Y, Chen Y, Yang L, Bian Z, Bragg F, Millwood IY, Mao E, Li Y, et al: Adiposity and risks of colorectal and small intestine cancer in Chinese adults: A prospective study of 0.5 million people. Br J Cancer 119: 248-250, 2018.

8. Erben V, Carr PR, Holleczek B, Stegmaier C, Hoffmeister M and Brenner $\mathrm{H}$ : Strong associations of a healthy lifestyle with all stages of colorectal carcinogenesis: Results from a large cohort of participants of screening colonoscopy. Int J Cancer 144: 2135-2143, 2019.

9. H Salim OE, Hamid HK, Mekki SO, Suleiman SH and Ibrahim SZ: Colorectal carcinoma associated with schistosomiasis: A possible causal relationship. World J Surg Oncol 8: 68, 2010.

10. Cheever AW: Schistosomiasis and colon cancer. Lancet 1: 1369-1370, 1981.

11. Xun Z and Su DL: Schistosoma japonicum and colorectal cancer: An epidemiological study in the People's Republic of China. Int J Cancer 34: 315-318, 1984.

12. Herman AM, Kishe A, Babu H, Shilanaiman H, Tarmohamed M, Lodhia J, Amsi P, Pyuza J, Mremi A, Mwasamwaja A, et al: Colorectal cancer in a patient with intestinal schistosomiasis: A case report from Kilimanjaro Christian Medical Center Northern Zone Tanzania. World J Surg Oncol 15: 146, 2017.

13. Hamid HKS: Schistosoma japonicum-associated colorectal cancer: A review. Am J Trop Med Hyg 100: 501-505, 2019.

14. Zanger P, Habscheid W, Kremsner PG and Dahm HH: Schistosoma japonicum infection and rectal carcinoid tumour: Underreported coincidence or neglected association? Epidemiol Infect 138: 1289-1291, 2010.

15. Katsidzira L, Gangaidzo IT, Makunike-Mutasa R, Manyanga T, Matsena-Zingoni Z, Thomson S, Matenga JA, Rusakaniko S and Ramesar R: A case-control study of risk factors for colorectal cancer in an African population. Eur J Cancer Prev 28: 145-150, 2019.

16. Drummond GB: Declaration of Helsinki. Anaesthesia 45: 59, 1990.

17. Sobin LH and Fleming ID: TNM classification of malignant tumors, fifth edition (1997). Union internationale contre le cancer and the American joint committee on cancer. Cancer 80: 1803-1804, 1997.

18. Bland JM and Altman DG: Survival probabilities (the Kaplan-Meier method). BJM 317: 1572, 1998.

19. Anonymous: Clinical and Laboratory Standards Institute (CLSI); CLSI releases guidelines for defining, establishing, and verifying reference intervals in the clinical laboratory. Biotech Week, 2008.

20. Carpelan-Holmström M, Louhimo J, Stenman UH, Alfthan H, Järvinen $\mathrm{H}$ and Haglund $\mathrm{C}$ : Estimating the probability of cancer with several tumor markers in patients with colorectal disease. Oncology 66: 296-302, 2004.

21. Bast RC Jr, Badgwell D, Lu Z, Marquez R, Rosen D, Liu J, Baggerly KA, Atkinson EN, Skates S, Zhang Z, et al: New tumor markers: CA125 and beyond. Int J Gynecol Cancer 15 (Suppl 3): S274-S281, 2005.

22. Chen MG: Assessment of morbidity due to Schistosoma japonicum infection in China. Infect Dis Poverty 3: 6, 2014.

23. Cao ZG, Li S, Zhao YE, Wang TP, Bergquist R, Huang YY, Gao FH, Hu Y and Zhang ZJ: Spatio-temporal pattern of schistosomiasis in Anhui Province, East China: Potential effect of the Yangtze River-Huaihe river water transfer project. Parasitolo Int 67: 538-546, 2018.

24. Wei Y, Huang N, Chen S, Chen D, Li X, Xu J and Yang Z: The diagnosis and treatment introspection of the first imported case of atypical cerebral schistosomiasis in Guangzhou city. PLoS Negl Trop Dis 12: e0006171, 2018.

25. Machida K, Yamada T, Shimura E, Machida K, Yamada T, Shimura E, Umemura M, Onoue S, Kaneko M, Mabuchi H, et al: Schistosomiasis japonica in a patient who emigrated from China: A case report. Nihon Shokakibyo Gakkai Zasshi 115: 1094-1100, 2018 (In Japanese).

26. Pearce EJ and MacDonald AS: The immunobiology of schistosomiasis. Nat Rev Immunol 2: 499-511, 2002. 
27. Tebeje BM, Harvie M, You H, Rivera V and McManus DP: T cellmediated immunity in CBA mice during Schistosoma japonicum infection. Exp Parasitol 204: 107725, 2019.

28. Fairfax K, Nascimento M, Huang SC, Everts B and Pearce EJ: Th2 responses in schistosomiasis. Semin Immunopathol 34: 863-871, 2012.

29. Zhu J, Xu Z, Chen X, Zhou S, Zhang W, Chi Y, Li W, Song X, Liu F and Su C: Parasitic antigens alter macrophage polarization during Schistosoma japonicum infection in mice. Parasit Vectors 7: 122, 2014.

30. Haeberlein S, Obieglo K, Ozir-Fazalalikhan A, Chayé MAM, Veninga $H$, van der Vlugt LEPM, Voskamp A, Boon L, den Haan JMM, Westerhof LB, et al: Schistosome egg antigens, including the glycoprotein IPSE/alpha-1, trigger the development of regulatory B cells. PLoS Pathog 13: e1006539, 2017.

31. Mostafa MH, Sheweita SA and O'Connor PJ: Relationship between schistosomiasis and bladder cancer. Clin Microbiol Rev 12: 97-111, 1999.

32. Wang M, Wu QB, He WB and Wang ZQ: Clinicopathological characteristics and prognosis of schistosomal colorectal cancer. Colorectal Dis 18: 1005-1009, 2016.

33. Dematei A, Fernandes R, Soares R, Alves H, Richter J and Botelho MC: Angiogenesis in Schistosoma haematobiumassociated urinary bladder cancer. APMIS 125: 1056-1062, 2017.

34. Filgueira NA, Saraiva CMA, Jucá NT, Bezerra MF and Lacerda CM: Schistosomal liver fibrosis and hepatocellular carcinoma-case series of patients submitted to liver transplantation. Braz J Infect Dis 22: 352-354, 2018.

35. Ma Y, Yang Y, Wang F, Zhang P, Shi C, Zou Y and Qin H: Obesity and risk of colorectal cancer: A systematic review of prospective studies. PLoS One 8: e53916, 2013.

36. Casimiro C: Etiopathogenic factors in colorectal cancer. Nutritional and life-style aspects. 2. Nutr Hosp 17: 128-138, 2002 (In Spanish).

37. Andres-Franch M, Galiana A, Sanchez-Hellin V, Ochoa E, Hernandez-Illan E, Lopez-Garcia P, Castillejo A, Castillejo MI, Barbera VM, Garcia-Dura J, et al: Streptococcus gallolyticus infection in colorectal cancer and association with biological and clinical factors. PLoS One 12: e0174305, 2017.

38. Chen C, Mao Y, Du J, Xu Y, Zhu Z and Chao H: Helicobacter pylori infection associated with an increased risk of colorectal adenomatous polyps in the Chinese population. BMC Gastroenterol 19: 14, 2019.
39. Darre T, Djiwa T, Dare S, Alassani F and Napo-Koura G: Difficult causality relationship between colorectal cancer and schistosomiasis. Pathol Oncol Res: Jan 2, 2019 (Epub ahead of print).

40. Schildgen V,Pieper M, Khalfaoui S, Arnold WH and Schildgen O: Human bocavirus infection of permanent cells differentiated to air-liquid interface cultures activates transcription of pathways involved in tumorigenesis. Cancers (Basel) 10: pii: E410, 2018.

41. Feng H, Lu AG, Zhao XW, Han DP, Zhao JK, Shi L, Schiergens TS, Lee SM, Zhang WP and Thasler WE: Comparison of non-schistosomal rectosigmoid cancer and schistosomal rectosigmoid cancer. World J Gastroentero 21: 7225-7232, 2015.

42. Decosse JJ, Ngoi SS, Jacobson JS and Cennerazzo WJ: Gender and colorectal cancer. Eur J Cancer Prev 2: 105-115, 1993.

43. Pengjun Z, Xinyu W, Feng G, Xinxin D, Yulan L, Juan L, Xingwang J, Zhennan D and Yaping T: Multiplexed cytokine profiling of serum for detection of colorectal cancer. Future Oncol 9: 1017-1027, 2013.

44. Nozoe T, Rikimaru T, Mori E, Okuyama T and Takahashi I: Increase in both CEA and CA19-9 in sera is an independent prognostic indicator in colorectal carcinoma. J Surg Oncol 94: 132-137, 2006.

45. Wang RF, Song BR, Peng JJ, Cai GX, Liu FQ, Wang MH, Cai SJ and Ye X: The prognostic value of preoperative serum CEA and CA19-9 values in stage I-III colorectal cancer. Hepatogastroenterology 61: 994-999, 2014.

46. Schistosomiasis and its prognostic significance in patients with colorectal cancer. National cooperative group on pathology and prognosis of colorectal cancer. Zhonghua Zhong Liu Za Zhi 8: 149-151, 1986 (In Chinese).

47. Wang M, Zhang YC, Yang XY and Wang ZQ: Prognostic analysis of schistosomal rectal cancer. Asian Pac J Cancer Prev 15: 9271-9275, 2014

48. Probst A, Schaller T, Ebigbo A and Messmann H: Colonic schistosomiasis and early rectal cancer: Coincidence or causal relationship? Endoscopy 46 (Suppl 1): UCTN: E671, 2014.

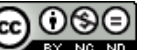

This work is licensed under a Creative Commons Attribution-NonCommercial-NoDerivatives 4.0 International (CC BY-NC-ND 4.0) License. 